\title{
Le refus d'aide et de soin dans les démences
}

\section{Refusal of assistance and care in dementia}

\author{
C. Racin ${ }^{a, b, *}$
}

a Hôpital Sainte-Périne, AP-HP, 11, rue Chardon-Lagache, 75016 Paris, France

${ }^{b}$ Laboratoire Subjectivité, Lien Social et Modernité (SuLiSoM - EA3071), Faculté de psychologie, université de Strasbourg, 12, rue Goethe, 67000 Strasbourg, France

Disponible sur Internet le 23 janvier 2019

\section{MOTS CLÉS}

Refus ;

Aide ;

Soin ;

Démence ;

Adaptation ;

EHPAD

\begin{abstract}
Résumé À partir d'une vignette clinique, nous montrons que la dénonciation des qualités objectives de l'objet, censé assurer les fonctions d'aide et de soin, doit être analysée au-delà des manifestations tangibles du refus. Celle-ci peut en effet trahir à la fois la fragilité du travail de figuration et de représentation psychiques, l'angoisse suscitée par la menace de perte de l'objet ainsi que les défenses mobilisées pour y faire face. Face au danger d'indifférenciation induit par la déliaison démentielle, l' « ancrage anal » et l' « expérience du miroir » sont proposés comme des opérateurs privilégiés du travail de care, à même d'étayer le travail psychique du suj et et de reconsidérer la question de l'adaptation à l'institutionnalisation des soins de longue durée.
\end{abstract}

\section{KEYWORDS}

Refusal;

Help;

Care;
Summary From a clinical vignette, we show that the denunciation of the objective qualities of the object, supposed to provide the functions of assistance and care, should be envisaged beyond the tangible manifestations of refusal. This denunciation can reflect the shortfalls of the work of figuration and psychic representation, the anguish caused by the threat of losing the

* Université de Strasbourg, Faculté de Psychologie, Laboratoire EA3071 SuLiSoM : Subjectivité, Lien social et Modernité, 12, rue Goethe, 67000 Strasbourg, France

Adresse e-mail : racin@unistra.fr 
Dementia; Adaptation; Nursing home object and the defence strategies mobilized to confront it. Faced with the danger of undifferentiation induced by dementia, "anal anchoring" and the "mirror experience" are proposed as privileged operators in the work of care, able to support the subject's psychic work and to reappraise the question of adaptation to long-term care.
S'intéresser aux conduites de refus dans les démences (exprimées verbalement ou manifestées dans des comportements) n'est pas sans comporter son lot de difficultés, tant ce phénomène apparaît comme un objet hétéroclite, polymorphe [1], aux contours mal définis qui charrie, dans les essais qui visent à le définir, le circonscrire et le comprendre, une imbrication de domaines et de voix parfois dissonantes. Les professionnels du soin gériatrique, qu'ils exercent à domicile, à l'hôpital ou en établissement d'hébergement, sont fréquemment confrontés à des situations de "refus " susceptibles de déranger, d'étonner, d'interpeller, d'embarrasser, de déconcerter, par leurs modalités singulières, et/ ou leur étendue, et/ou leur intensité, jusqu'à parfois désavouer ces professionnels dans leurs missions d'aide et de soin, et confiner les proches à l'inquiétude et à l'impuissance.

Dans les démences, d'autant plus lorsqu'un diagnostic a été posé, le «jugement d'incapacité » ou " trouble du jugement » apparaît souvent mis en avant pour expliquer un phénomène dont la signification et la valeur de message sont susceptibles d'échapper. Dans le soin gériatrique, c'est l'ombre d'un paradigme déficitaire qui tombe sur l'objet du refus du suj et dément, qui conduit parfois trop rapidement à une compréhension rabattue sur les registres symptomatologiques et psychopathologiques du déni ou de I'anosognosie, dont les effets d'assignation négative et réifiante ne sont pas à négliger. Le procès de subjectivation qui met en doute les capacités du sujet à s'énoncer tend en effet à prescrire une place au sujet âgé, susceptible de précéder la perception et d'anticiper la qualification, par les professionnels, les proches et le sujet lui-même, de la valeur de message contenue dans ces refus. La compréhension de ces phénomènes de refus les fait souvent apparaître comme une notion descriptive dépeignant un mode de relation particulier du sujet avec son monde, déterminé par une faillite du raisonnement et du jugement qui compromettrait toute possibilité de percevoir les bénéfices de l'aide proposée. Néanmoins, autant il est important de ne pas négliger les difficultés cognitives qui restent à évaluer sous cet angle, autant ces difficultés ne disent rien de ce que vit le suj et ou de la façon dont il perçoit I'aide qui lui est proposée. S'en tenant à cette seule référence indexée à un critère de fiabilité ou de validité du raisonnement, cette conception ferait courir le risque de connoter négativement et réduire fâcheusement la question du refus à un modèle psychopathologique (neurologique ou psychiatrique). Une approche plus féconde pour l'accompagnement thérapeutique semble pouvoir être trouvée si nous reconnaissons, dans les conduites de refus, une modalité du lien à autrui chargée d'un contenu latent et $d^{\prime}$ un contenu manifeste. Dans le souci de rester aussi proche que possible de la diversité de la réalité clinique et de la polysémie des situations de refus, il s'agirait dès lors d'en spécifier les formes cliniques et psychopathologiques et leurs inflexions éventuelles au cours d' un processus démentiel.

\section{Cas clinique}

M. B. 85 ans - rencontré dans le cadre d'une hospitalisation pour une chute, dans un contexte de perte d'autonomie liée à une maladie d'Alzheimer évoluant à un stade "modéré »- offre une illustration clinique qui, à défaut de rendre compte de la multiplicité des expériences, de qualifier l'hétérogénéité de ces vécus, fournit une opportunité d'examiner la place et le sens que le refus est susceptible d'occuper dans l'économie psychique d'un sujet. II s'agit d'un exemple de refus d'aide et de soin dans le cadre d'un projet d'institutionnalisation amorcé à I'hôpital. Dans le cadre d'une étude longitudinale, nous nous sommes intéressée aux mouvements complexes du fonctionnement psychique suscités par l'annonce du projet d'institutionnalisation d'une part, et à leurs effets sur le devenir du sujet âgé institutionnalisé d'autre part, que ce devenir se déroule au domicile ou en établissement d'hébergement pour personnes âgées dépendantes (EHPAD). Autour de ce moment de "crise ", le refus apparaît souvent comme le négatif du "consentement » (ou de I' " assentiment »), entraînant une tension conflictuelle entre le mandat de soin et le mandat de protection confiés aux professionnels (en référence aux nombreux débats qui entourent les questions du respect de l'autonomie et de la liberté du sujet âgé dément).

Lorsque nous rencontrons M. B. au cours de son hospitalisation, un sentiment d'incompréhension domine son discours, qui semble dans un premier temps traduire un mouvement d'opposition à ce qui fait l'effet d'une interprétation sauvage. Cet effet n'est pas sans lien avec le sentiment d'inquiétante étrangeté qui surgit de la confrontation au miroir offert par cette "scène " hospitalière, dans lequel il découvre une image d'un autre lui-même qui rompt l'illusion de complétude et d'illimité. Placé face à son reflet dans le miroir, M. B. ne reconnaît pas seulement son image actuelle ; il est également confronté à la perception ponctuelle de l'anticipation d'un corps qui échappe irréductiblement aux tentatives de maîtrise du travail de la mort en 
soi, brèche ouverte à la manifestation de la précarité temporelle de son être, qu'il exprime à travers un sentiment d'impuissance face à la difficile confrontation à la souffrance de ses pairs dans le service, miroir qui lui renvoie par ailleurs l'image menaçante d'un devenir inquiétant : "Moi aussi j'ai déjà appelé au secours, vous savez. Vous le faites sans y penser. [...] Moi j'ai toujours été actif, j'ai toujours tout obtenu par moi-même tout le temps, j'ai toujours été indépendant ».

M. B. se décrit avec fierté comme «autodidacte», pourvu $d^{\prime}$ ' ingéniosité » et d'un " tempérament passionné », ce qui lui a permis de mener une "belle carrière » et $d$ 'assumer des responsabilités. La garantie de sa «virilité », qui occupe une place importante dans ses préoccupations, ne tient pas tant aux performances du corps, nous dit-il, qu'à l' « intelligence » du corps, à son "habileté », qui lui permet de vérifier dans la réalité les hypothèses qu' une curiosité insatiable ne cesse de lui souffler. M. B. nous en donne immédiatement un exemple, non sans une certaine jubilation, en exposant de quelle manière il a résolu le problème, insoluble pour les soignants à l'hôpital, d'une lampe défaillante qui n'assurait plus ses fonctions. "Je suis habile de mes bras et de mes jambes ", affirme-t-il, tout en constatant douloureusement: «Je me rends quand même compte que je ne suis plus capable de faire maintenant ce que je faisais il y a deux mois. Vous voyez, tout vous coûte, tout pose problème. Et puis je ne marche pas ». À l'hôpital, M. B. réalise qu'il n'y a " pas de jeunes » et s'en étonne, lui qui ne se sent «pas âgé », mais " handicapé ». Privé de certaines capacités qui lui permettaient toujours de « dépanner » les uns et les autres, qui lui valaient la reconnaissance de son entourage, M. B. redoute aujourd' hui une perte de maîtrise sur les choses qui le concernent. II se sent "inutile " et s'évertue à retrouver son indépendance, dont cette ingéniosité constitue pour lui le ressort incontournable. II aimerait rentrer chez lui, mais dans des conditions qui lui permettent d'assurer une continuité avec les investissements antérieurs, et notamment de garantir la pérennité des liens avec ses enfants, petits-enfants et voisins. De sorte que son domicile doit, dans sa perspective, refléter l'image d'un homme actif, indépendant, capable de porter assistance et de soutenir des "échanges intéressants", ce qui lui semble incompatible avec les propositions d'aménagement de son rez-de-chaussée auxquelles est conditionné son retour (qui impliquent notamment l'installation d'un lit médicalisé dans le salon)

Nous retrouvons $M$. B. quatre mois après sa sortie de I'hôpital dans I'EHPAD où il a emménagé. II a connu encore quelques épisodes de fragilisation somatique et s'adapte-aux dires des soignants - difficilement à ce nouveau mode de vie. Les aides et différents soins semblent vécus dans la contrainte. II exprime par exemple régulièrement son refus d'être touché lors des soins d'hygiène et son agacement à se sentir privé de la gestion de ses "affaires », sentiment exacerbé par un fonctionnement institutionnel qui promeut la présence systématique d'un soignant pour s'assurer du bon déroulement des gestes de la vie quotidienne. La tension entre autonomie et dépendance vient s'actualiser dans des conflits interpersonnels et dans des conduites d'opposition avec les professionnels de santé jusqu'à conduire, aux dires des soignants, à des situations d'impasse qui ont pu favoriser des passages à l'acte, à l'instar de l'imposition de certaines modalités de réalisation des gestes de la vie quotidienne en dehors d'une décision partageable en équipe. Ses tentatives de maîtrise, par exemple lorsqu'il conserve son linge pour réaliser lui-même sa lessive, donnent parfois lieu à des rapports de force avec certains soignants, jusqu'à susciter chez lui une insécurité qui se traduit par des impressions de dépossession et de vol.

M. B. tend à conférer une fonction exclusivement « utilitaire » à l'institutionnalisation. Cette dynamique promeut sur le plan conscient un investissement des soignants qui serait essentiellement déterminé par les nécessités inhérentes au maintien de l'autoconservation, en faisant porter l'accent sur les traits dont l'objet doit être affecté pour remplir ce rôle (le "professionnalisme » des aides, leur " habileté » et leur " efficacité » sont ainsi mis en avant), tout en dénonçant la faillite des fonctions attendues. Cependant, le procès de fiabilité régulièrement adressé à l'environnement par certains sujets âgés déments, tout comme l'incrimination des qualités objectives de l'objet externe sur lequel il faudrait désormais pouvoir compter, ne doit pas nous faire oublier combien ces mouvements n'en restent pas moins, en définitive, toujours infiltrés du dévoilement ou de la dénonciation de la fragilité du sentiment de sécurité interne, et en cela, de la faillite possible des objets internes. M. B. dénonce un environnement extrêmement contraignant et par ailleurs insuffisamment soutenant, au sein duquel une présence chaleureuse et impliquée fait cruellement défaut. En dépit de nos invitations à en parler, les soignants semblent peu investis par M. B., anonymisés dans leur identité et dans leurs intentions ( « on ne sait jamais qui est qui, ni ce qu'ils viennent faire »), et donnent lieu à la rencontre renouvelée avec un environnement inconnu et inquiétant.

\section{Discussion}

\section{À propos de l'insistance portée à la fonction utilitaire de l'institutionnalisation}

L'insistance portée à la matérialité des pratiques mobilisées dans et par le care, loin de cantonner les objets du care (nous faisons référence ici aux pourvoyeurs d' aide et de soin) à leurs seules qualités objectives, nous permet d'entendre aussi que l'objet en question est un objet " seulement dedans, aussi dehors " - selon I'heureuse expression de Botella et Botella [2] - un objet externe dont la perception soutient le travail de représentation. En ce sens, le refus de soin peut, en certaines circonstances, traduire une mobilisation défensive contre l'angoisse de perte de l'objet, ou encore comme le "refus de l'irreprésentable de l'objet » [3], chez un sujet en prise avec un processus de déliaison démentielle menaçant la continuité psychique et les capacités de différenciation. L'insistance, par ailleurs, portée à ce registre de la matérialité, semble traduire l'urgence de sécuriser le présent pour étayer la continuité du sentiment d'exister, en écartant en apparence les questions relatives à la poursuite du désir. Face à ce qui peut être 
repéré comme une injonction paradoxale véhiculée par le projet d'institutionnalisation qui propose, en réunissant les incompatibles, de continuer à vivre tout en rendant la mort possible, une solution plus ou moins opérante, plus ou moins durable, semble être trouvée chez certains sujets dans un recours au clivage qui permet de maintenir l'unité du Moi tiraillé entre deux tendances. Cette mobilisation défensive soutient la séparation du corps érotique et du corps du besoin, dès lors "libéré » de ses revendications pulsionnelles, libidinales et agressives, tout comme elle soutient la séparation de l'objet du besoin et de l'objet du désir. De ce fait, elle tient à distance la confrontation à un autre qui possède ses exigences propres, poursuit ses désirs personnels et ne se borne pas volontiers à réaliser ceux du sujet, voire même les frustre ou les menace. Par le clivage, la (re)connaissance de l'altérité - qui porte au plus haut point sur le sexuel et sur la mort - est susceptible de coexister avec une méconnaissance qui en dissout les effets. Dès lors, doit-on reconnaître dans ce déni d'altérité l'empreinte patente d'un échec du travail psychique en situation de dépendance (ou «travail de dépendance ») ? II semble intéressant d'entendre aussi ce en quoi la centralité occupée par le pôle autoconservatif peut venir traduire la fragilité des ressources défensives habituelles, et la nécessité de recourir au clivage pour faire face à la résurgence aiguë du besoin de continuité.

\section{Dans l'ombre des conduites de refus: les malentendus de I' « adaptation » à l'institutionnalisation}

Pas d' « adaptation réussie » donc du côté de M. B. ? Car s'il y a un aspect susceptible d'apparaître comme le « négatif » du « refus», c'est bien la question de l' « adaptation », adaptation pouvant avoir de multiples aspects : celui de I'« adaptation-transformation » qui intègre la nouveauté ambivalente, à l'horizon de laquelle le sujet continue à se projeter dans l'à-venir, à développer ses aspirations, à déplacer ses investissements vers des sources de satisfaction inédites qui étayent la révision des idéaux sous l'impact de la réalité ; celui de l' « adaptation-retrait » qui obture I'horizon, signe l'échec de l'inscription de l'expérience, la chronicisation de la situation de crise, le déni du changement vécu ou la précipitation dans la rupture; celui de I' « adaptation-conformisme » qui concourt à supporter sans se plaindre, véritable création contraphobique confinant au "stoïcisme muet », qui vise à atténuer l'impact des excitations via l'inhibition, la répression et la contention des affects. Lorsque les aspects défensifs du fonctionnement psychique sont ainsi mis au service d'une soumission excessive, la pression conformiste constitue un ressort inquiétant de ce que l'on pourrait appeler, en empruntant cette formulation à Triandafillidis [4], le mythe d'une " normalité fictive » dans le grand vieillissement. Pseudo-réussite adaptative donc... mais à quel prix, quand l'investissement contraint des objets du care, étouffant le feu de la contestation mais excluant, du même coup, le champ du désir, fait apparaître des sujets bien ternes, éteints, passant « inaperçus»?

Pourtant, M. B. a pu développer quelques liens dans I'établissement, soutenus par sa participation régulière aux activités proposées. Lorsque nous le retrouvons, $M$. B. ne se souvient pas de nos rencontres précédentes, mais accepte volontiers notre présence. La relation est d'emblée teintée de l'investissement libidinal de M. B., qui se montre séducteur à notre égard, nous accompagnant fièrement dans sa chambre, «en tout bien tout honneur » prend-il soin de préciser, usant de maints détours pour nous faire visiter les lieux, cette manœuvre semblant tout à la fois s'ingénier à dissimuler ses difficultés à se repérer dans l'établissement, qu'à nous exhiber, espiègle, au regard curieux et scrutateur des autres résidents.

En même temps, M. B. n'a de cesse d'agiter les atouts qui lui permettent de se tenir érigé face à la menace, dans un mouvement qui trahit cependant la précarité de ces appuis à valence de réassurance phallique-narcissique : d'après lui, il peut s'habiller seul, mais de nombreuses affaires « vitales» sont manquantes (en fait, il ne les retrouve pas) ; il peut ranger "son intérieur », mais les meubles sont insuffisants (il oublie où il a rangé ses affaires), et son plaisir du classement, soutenu antérieurement par ses " prédispositions », son caractère " méthodique », n'est plus aussi assuré aujourd' hui, ce qui lui fait dire : « je n'ai plus de repères ici ", et que peut-être il lui faudrait une femme pour l'aider. "Mais, ils, ils sont partis du principe que moi, j'étais dépendant parce que j'étais un homme seul. Ah ben non, dans tous les cas, je, si je suis ici, c'est parce que je suis seul. Je ne... je ne... je n'ai pas, je n'ai aucune, physiquement, je n'ai eu aucune raison de me trouver là. Vous voyez ? ». Au milieu de ce monde de "vieux » dont les plaisirs sont réduits à des voies de satisfaction régressives, I'atout suprême, imposant sa supériorité à tous les autres, est celui auquel M. B. donne le nom d' "indépendance ", cette indépendance étant devenue, à l'épreuve du narcissisme des petites différences $[5,6]$ en EHPAD, la vertu de ceux qui "peuvent se déplacer » et "monter les escaliers ", sans avoir besoin d'utiliser un fauteuil roulant ou d'attendre I'ascenseur. La revendication phallique se polarise désormais moins sur les capacités intellectuelles, comme c'était le cas à l'hôpital, que sur la mobilité (dans une visée probablement défensive contre la perception de ce qui se délite par ailleurs), qui lui permet de s'ériger et de se porter où bon lui semble, alors que les autres sont cloués sur place, immobilisés et castrés dans leur moyen d'action.

Les destins de la relation d'aide, son évolution dans le temps, dépendent en conséquence étroitement des virtualités trophiques ou des inquiétudes issues de la résonance entre les caractéristiques singulières de fonctionnement du sujet et un environnement qui possède ses particularités propres, et notamment une plus ou moins grande plasticité pour se laisser « utiliser » et « déformer » un tant soit peu, à I'appui d'une contribution masochique minimale. Pour peu, également, que l'on accorde toute la valeur et l'attention nécessaires à ce qui se joue là, à savoir le profond sérieux du sujet qui croit créer ce qu'il trouve [7], souvent à partir de ces « je-ne-sais-quoi » et ces «presque-rien» dont J ankélévitch [8] fait des choses d'autant plus importantes qu'elles sont impalpables, invisibles et manipulables. À cette condition peut-on espérer que le suj et âgé dément n'en finit pas $d^{\prime}$ inventer ses objets d'amour, que loin d'être contraint à I'issue désespérante du «subir » et du "pâtir », il continue à manipuler, à fabriquer, à «se » façonner un monde, à rêver 
sous le regard d'autrui un projet par lequel il peut soutenir un désir qui le prolonge.

\section{Perspectives thérapeutiques: « ancrage anal » et « expérience du miroir», vecteurs privilégiés du travail psychique en situation de démence}

L'accent que nous venons de porter au registre du «faire » ( " manipuler », " fabriquer », " façonner ») et à la question du regard nous invite à souligner le rôle de l'analité et de l'expérience du miroir dans la construction par le sujet de son rapport au temps, et dans la constitution continue d'une image de soi à venir. Ce qui nous intéresse particulièrement, c'est d'examiner la manière dont les dispositifs de soins de longue durée, proposés au domicile ou en EHPAD, parviennent ou non à favoriser un travail de care prompts à soutenir un ancrage anal du travail psychique en situation de démence, par lequel le sujet expérimente le plaisir de la maîtrise, source de réapprovisionnement narcissique face à ce qui semble se déliter par ailleurs. Cette " obsessionnalisation » du care joue un rôle essentiel en étayant avantageusement une efficacité du contrôle et une fragmentation des affects. Recours fort précieux qui protègent le sujet des aléas des circonstances relationnelles, tout en lui permettant d'éprouver un sentiment de fierté et de valeur personnelle, à partir d'une reconnaissance qui porte sur le «faire ». À cet endroit, le «faire » dont il est question ne se confond nullement avec le «faire " productif et compétitif rehaussé par le culte de la performance inscrit dans nos sociétés, stimulant les défenses phalliques viriles; il concerne ici le «pouvoir faire » par lequel le sujet exprime son "mode d'être au monde " à travers des réalisations concrètes et figuratives, à la manière dont l'orfèvre sculpte la matière précieuse, réalisations dont des bénéfices narcissiques et/ ou sublimatoires peuvent être escomptés. L'opérativité de cette fonction tient évidemment dans la souplesse de ses aménagements, qui exige de ne pas "confondre "emprise", (simple besoin de domination violente de l'objet) avec la "maîtrise" qui implique une intériorisation objectale possible avec un plaisir découlant de cette façon d'utiliser le rapport à l'objet »[9]. L'importance d'une reconnaissance portant sur le « faire » souligne les enjeux de transmission dans la relation de care, transmission des "savoir-faire » de l'un pour favoriser les «pouvoir-faire » de l'autre, par lesquels se maintiennent les assises narcissiques.

Reconnaissance qui met à l'épreuve la capacité du sujet dément in care à « recevoir par voie symbolique [...] anale des apports narcissiques précieux d' origine extérieure et de les conserver en lui, en procédant à leur intégration identificatoire, narcissique d'abord, sans se sentir dans l'obligation de rejeter automatiquement ce qu'il reçoit » [9]. Encore faut-il, en suivant toujours les développements de Bergeret [9], que le narcissisme des pourvoyeurs du care soit « préalablement suffisant pour que ce don puisse se réaliser sans sentiment de perte, d'angoisse, voire de dépossession narcissique dramatique ».

Reconnaissance qui repose sur la singularité saisie dans la quotidienneté, reconnaissance susceptible d'être éprouvée à travers les gestes les plus ordinaires du quotidien, là où
I'attention portée à autrui, la sollicitude qui pousse à vouloir le bien d'autrui, conduit aisément à se focaliser sur les incapacités et à imposer nombre de contraintes au nom du "prendre soin ».

Reconnaissance qui repose sur l'inventivité quotidienne pour présenter des objets transitionnels qui puissent progressivement, processuellement, être utilisés dans l'espace intermédiaire du care, objets cocréés-trouvés à l'appui desquels relancer des investissements, là où, à ne $s^{\prime}$ en tenir qu'à l'évaluation des conduites manifestes des refus, le risque est grand de précipiter des habitudes de soin qui se réfugient dans la tentative de maîtrise d'une souffrance psychique, maîtrise qui passe malheureusement encore trop souvent par la voie de la prescription médicamenteuse.

\section{Conclusion}

Les manifestations souvent déroutantes du refus d'aide et de soin du sujet dément attisent régulièrement la tentation à une objectivation qui viserait à en maîtriser les effets. La valeur défensive de cette conduite mérite d'être soulignée tant elle peut, dans un premier temps, favoriser la réduction de l'angoisse liée à l'incertitude. Néanmoins, la clinique quotidienne montre combien la compréhension des réactions conflictuelles du sujet aux propositions d'aide et de soin qui lui sont faites, s'immisçant dans les «petits riens " de la vie quotidienne, trouve toute sa fécondité dans la mise en partage et en travail au sein des temps de réunion en équipe. L'espace psychique élargi que peut représenter, dans des circonstances favorables, ce temps d'élaboration collective, favorise la réflexion sur la forme d'opérationnalisation du care susceptible d'être re-saisie par le sujet. Un care inassimilable au " cela va de soi », un care qui ne se décrète pas du seul fait de l'obligation morale que nous incombe la confrontation à la vulnérabilité d'autrui, et pour lequel des voies d'activation doivent être recherchées, souvent par tâtonnements successifs et improvisation. Suivant cette perspective, l'activation du care ne peut être contenue dans les impératifs procéduraux prescrits par le haut qui font florès dans les institutions gériatriques, lorsqu'ils négligent le particularisme indissociable de l'effectivité du care. Car c'est en puisant dans l'expérience vécue, nécessairement réfléchie par le regard d'un autre, que le care tire ses conditions de possibilité.

\section{Déclaration de liens d'intérêts}

L'auteure déclare ne pas avoir de liens d'intérêts.

\section{Références}

[1] Balard F, Somme D. Le refus d'aide et de soin des personnes âgées en situation complexe. Nouvelles pratiques sociales 2011:24(1):85-100.

[2] Botella C, Botella S. La figurabilité psychique (2001). Paris: In Press; 2007

[3] Guentcheff I. Le refus de soin du sujet en démence : une réflexion sur le terme d'opposition. Neurol Psychiatr Geriatr 2016; 16(93): 133-5. 
[4] Triandafillidis A. Stratégies d'immortalité. Adolescence 2010; 72(2):443-60.

[5] Freud S. Le tabou de la virginité (Contributions à la psychologie de la vie amoureuse III) (1917). In: Fuvres complètes XV (1916-1920). Paris: Presses Universitaires de France; 2006. p. 77-96.

[6] Freud S. Psychologie des masses et analyse du moi (1921). In: Fuvres complètes XVI (1921-1923). Paris: Presses Universitaires de France; 2010. p. 1-83.
[7] Winnicott DW. Jeu et réalité. L'espace potentiel (1971). Paris: Gallimard; 2002

[8] Jankélévitch V. Le J e-ne-sais-quoi et le Presque-rien. Paris: Seuil; 1980.

[9] Bergeret J. L' « analité » et la maîtrise. Revue Française de Psychanalyse 1995; 59(3):659-82. 